mortality counts using adjusted rates for national and regional DuPont worker populations. Robust specification of priors will be sought. Implementation of the calculations will be developed in common software.

Conclusions We plan to develop a method for SMR calculation that accounts for the healthy worker selection effect both in the point estimate and uncertainty interval.

\section{NAPPING DURING NIGHT SHIFT AND SELF-REPORTED HYPERTENSION AMONG NURSING WORKERS}

'Lucia Rotenberg, 'Aline Silva-Costa, 1,2Paulo Roberto Vasconcellos-Silva, 'Rosane Harter Griep. 'Oswaldo Cruz Foundation, Rio de Janeiro, Brazil; ${ }^{2}$ Universidade Do Rio de Janeiro-Unirio, Rio de Janeiro, Brazil

\subsection{6/oemed-2014-102362.377}

Objectives Night and shift work are suggested risk factors for hypertension. Considering the relationship between sleep deprivation and blood pressure, this study focuses on self-reported hypertension and napping during night shift. Our aims are (1) to analyse the prevalence of hypertension among day and night workers and (2) to test the association between napping regularly during night shifts and prevalence of hypertension among night workers.

Method This cross sectional questionnaire study was carried out at 18 public Brazilian hospitals in 2010-2011 ( $\mathrm{N}=3229$ registered nurses). Only women workers were included in the analysis $(\mathrm{N}=1992)$. Statistical treatment of data was carried out in two steps: (i) assessing self-reported hypertension considering work schedule and (ii) analysing nap habits during night shifts and self-reported hypertension.

Results Mean age was $39.9(\mathrm{SD}=10)$ years. Napping during the night shift (for up to three hours) increased the odds of self-reported hypertension 1.8-fold (95\% CI 1.36-2.45) compared with day workers with no experience on night shifts, after adjusting for age, physical activity, smoking habits, and housework. Among night workers, sleeping during the night shift reduced the odds of reporting hypertension $(\mathrm{OR}=0.79 ; 95 \% \mathrm{CI} 0.63-1.00)$, compared to those who reported not to sleep during the night shifts.

Conclusions The higher prevalence of hypertension among shift workers was confirmed. Dipping patterns and blood pressure control may be influenced by short periods of sleep in night shifts. The potential positive effect of naps on blood pressure deserves further investigation through automatic monitoring.

\section{COUNTERFACTUALS, QUANTUM MECHANICS AND G- ESTIMATION: CAUSALITY THREATENS EPIDEMIOLOGY}

1,2Peter Morfeld. 'Institute for Occupational Epidemiology and Risk Assessment of Evonik Industries, Essen, Germany; ${ }^{2}$ Institute and Policlinic for Occupational Medicine, Environmental Medicine and Preventive Research, University of Cologne, Cologne, Germany

\subsection{6/oemed-2014-102362.378}

Objectives Causal reasoning should have an explicit formal structure.

Method Such a structure can be provided with the help of counterfactuals. This approach allocates different versions (factual and non-factual) of exposures and responses to every basic study unit (e.g., a subject observed at one point of time). Comparisons of these versions within the unit imply causal statements about the effect of exposures. This approach may appear unusual and strange but it is consistent to basic principles of modern physics (superposition principle of quantum mechanics).

Results The outline of causality in counterfactual terms is helpful to solve problems like defining and measuring direct and indirect causal paths or to specify biases and adjusting procedures. In contrast to experimental research observational studies (like those performed in epidemiology) suffer from missing randomization. A causal concept is important to understand the reliability of such studies: a strict counterfactual framework motivates to analyse observational studies in terms of generalised treatments (“G”). G-estimation is a procedure that defines the causal effect estimates on the individual level by counterfactual failure times. Causal models are nested within estimating models ("structurally nested failure time models").

Conclusions Such a strict counterfactual reasoning challenges standard estimators and estimating procedures usually applied in epidemiology.

\section{THE HEALTHY WORKER SURVIVOR EFFECT DISSECTED: ADDRESSING COMPONENT PARTS}

${ }^{1}$ Jonathan Chevrier, ${ }^{2}$ Dan Brown, ${ }^{2}$ Sally Picciotto, ${ }^{2}$ Sadie Costello, ${ }^{2}$ Andreas Neophytou, ${ }^{2}$ Ellen Eisen. ${ }^{1}$ Mc Gill University, Montreal, Quebec, Canada; ${ }^{2}$ University of California, Berkeley, Berkeley, CA, USA

\subsection{6/oemed-2014-102362.379}

Objectives The healthy worker survivor effect (HWSE) is a wellrecognised bias usually described as a form of selection bias or confounding. A more precise epidemiologic explanation, however, has been elusive. We distinguish several components of the HWSE and suggest methods for bias correction in occupational cohort studies.

Method Although generally referred to a single effect, we demonstrate using simulation studies that there are in fact four distinct aspects of the HWSE. Two aspects, (1) time-varying confounding by variables on the causal pathway and (2) heterogeneity in susceptibility, are functions of the underlying process of the exposure and disease under study. The other two, (3) left truncation and (4) right truncation, are functions of how the data are collected, ie the study design. We quantify the bias induced by each aspect of HWSE on dose-response parameter estimates and apply methods designed to reduce the bias.

Results We find that causal techniques, eg, g-estimation and IPTW, can correct for time-varying confounding. Heterogeneous susceptibility in combination with either left or right truncationcan be corrected using inverse probability of censoring weights. The health related variables needed to make either of these methods succeed in reducing the bias are often unmeasured.

Conclusions HWSE occurs due to the presence of any of four factors that may function separately or in concert to produce a downward bias if not accounted for. We provide guidance for methodologic approaches to reduce the bias.

\section{G-ESTIMATION: WHY DOES IT WORK AND WHAT DOES IT OFFER?}

${ }^{1}$ Sally Picciotto, ${ }^{1}$ Ellen A Eisen, ${ }^{2}$ Jonathan Chevrier. ${ }^{1}$ University of California, Berkeley, CA, USA; ${ }^{2}$ McGill University, Montreal, PQ, Canada

10.1136/oemed-2014-102362.380 